\title{
Towards the measurement of carbon and oxygen spectra in cosmic rays with DAMPE
}

\author{
Libo Wu, ${ }^{a b d e, *}$ Mingyang Cui, ${ }^{c}$ Dimitrios Kyratzis, ${ }^{d e}$ Andrea Parenti ${ }^{d e}$ and Yifeng \\ Wei $^{a b}$ on behalf of the DAMPE ${ }^{\dagger}$ Collaboration \\ (a complete list of authors can be found at the end of the proceedings) \\ ${ }^{a}$ State Key Laboratory of Particle Detection and Electronics, University of Science and Technology of \\ China, Hefei, Anhui 230026, China \\ ${ }^{b}$ Department of Modern Physics, University of Science and Technology of China, Hefei, Anhui 230026, \\ China \\ ${ }^{c}$ Key Laboratory of Dark Matter and Space Astronomy,Purple Mountain Observatory, Chinese Academy \\ of Sciences, Nanjing 210023, China \\ ${ }^{d}$ Gran Sasso Science Institute (GSSI), Via Iacobucci 2, I-67100 L’Aquila, Italy \\ ${ }^{e}$ Istituto Nazionale di Fisica Nucleare (INFN) -Laboratori Nazionali del Gran Sasso, I-67100 Assergi, \\ L'Aquila, Italy \\ E-mail: libo.wu@gssi.it, mycui@pmo.ac.cn, dimitrios.kyratzis@gssi.it, \\ Andrea.Parenti@gssi.it, weiyf@ustc.edu.cn
}

The space experiment DArk Matter Particle Explorer (DAMPE) is developed and supported by an international collaboration including institutes and universities from China, Italy and Switzerland, DAMPE is designed to measure cosmic-rays (CRs) and gamma-rays in space, and since December 2015 it is collecting data with smooth and continuous operations. The instrument consists of two layers of plastic scintillators to identify the charge of individual elements from proton to iron, a silicon-tungsten tracker providing accurate particle tracking and complementary charge measurement, and a thick calorimeter (32 radiation lengths) providing a good potential to measure CRs in the energy range from $50 \mathrm{GeV}$ up to $100 \mathrm{TeV}$. This measure is of fundamental importance to shed light on the mechanism of acceleration and propagation of cosmic rays in the Galaxy. In this report, the selection criteria of the carbon and oxygen components will be illustrated; Some comparison of charge between Monte Carlo data and flight data have been done. And efficiency of event selections also be validated by Monte Carlo simulations.

$37^{\text {th }}$ International Cosmic Ray Conference (ICRC 2021)

July 12 th - 23rd, 2021

Online - Berlin, Germany

\footnotetext{
*Presenter

${ }^{\dagger}$ The DAMPE mission is funded by the Strategic Priority Science and Technology Projects in Space Science of the Chinese Academy of Sciences. This work is supported by the National Key Research and Development Program of China (2016YFA0400200 and 2016YFA0400202), the Project supported by the Joint Funds of the National Natural Science Foundation of China (No. U1738208, U1738139 and U1738135) and the National Natural Science Foundation of China (No. 11673021 and No. 11705197). In Europe the activities and the data analysis are supported by the Swiss National Science Foundation (SNSF), Switzerland; the National Institute for Nuclear Physics (INFN), Italy.
} 


\section{Introduction}

Many space-borne experiments, such as AMS-02 [1], PAMELA [2], CALET [3], Fermi-LAT [4], measured galactic CR fluxes which are important in understanding their origin and propagation mechanisms. DAMPE is a satellite-based general-purpose high energy particle detector. It was launched into a sun-synchronous orbit around the earth at an altitude of $500 \mathrm{~km}$ on December 17, 2015. The scientific objectives of DAMPE are the searches for Dark Matter decay or annihilation signatures, gamma-ray astronomy and the measurement of CR fluxes. Thanks to the great thickness of the DAMPE calorimeter and large acceptance, the satellite collected many CR events for more than five years. In this work we present the analysis procedure for carbon and oxygen fluxes and specifically the selection criteria adopted to properly identify them.

\section{DAMPE detector}

The DAMPE detector [5, 6] is composed of the following sub-detectors (see Fig. 1): a Plastic Scintillator strip Detector (PSD) [7], a Silicon-Tugsten tracKer (STK) [8], a BGO imaging calorimeter [9], and a NeUtron Detector (NUD). The PSD is designed to measure the charge of incident particles up to $Z=26$ and provides an anti-coincidence veto signal for gamma rays. The STK is dedicated to reconstruct the trajectories of charged particles and also to provide electrongamma identification. The BGO calorimeter, which has a depth of 32 radiation lengths and 1.6 nuclear lengths, is in charge for CR energy measurement and provides a powerful electron-hadron discrimination. The NUD mainly improves the electron-proton separation power, especially for energy above $\mathrm{TeV}$.

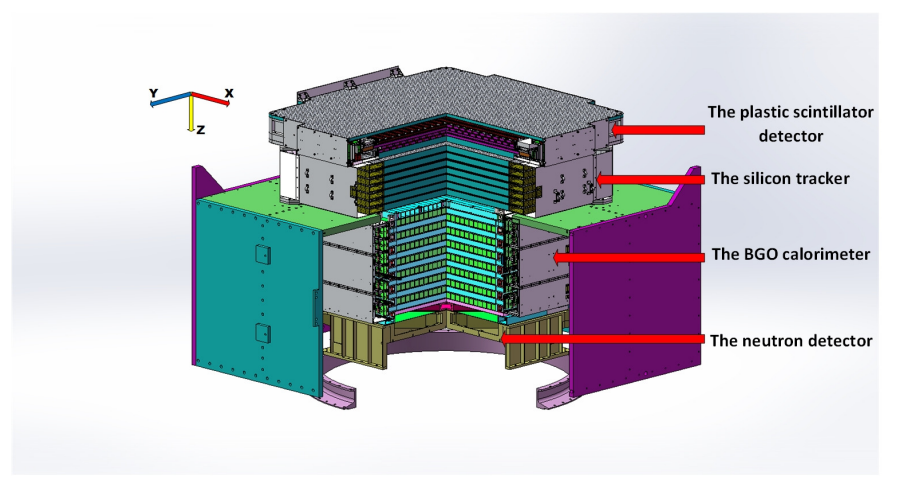

Figure 1: Side view of the DAMPE detector.

\section{Data analysis}

The data used for the analysis described in this work were taken from January $1^{s t}, 2016$ to December $31^{s t}, 2020$ (Fig. 2). The number of collected events per day is about 5 millions, and the data taking mode of DAMPE is very stable. After excluding the lost time, such as the time in South Atlantic Anormaly (SAA) region, the instrumental dead time (3.0725 ms per event) and the time for on-orbit calibration of the sub-detectors, the residual fraction of live time is about $76.19 \%$. For 
Monte Carlo(MC) data, Geant4 is the main simulation engine for DAMPE experiment. The model of Geant4 used for this analysis is FTFP_BERT(Hadronic Model) with a hemispherical source of $1.0 \mathrm{~m}$ radius. The energy range of $\mathrm{MC}$ data is from $50 \mathrm{GeV}$ to $100 \mathrm{TeV}$.

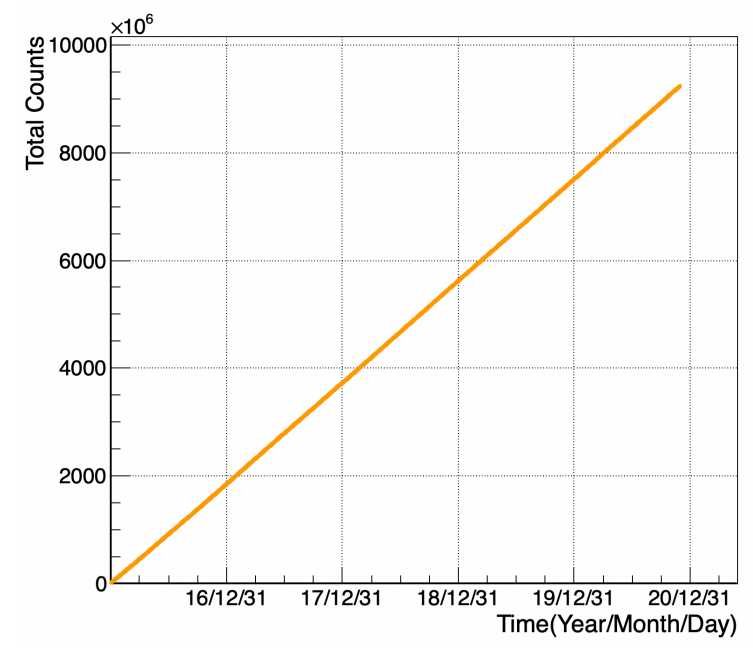

Figure 2: Event rate recorded by DAMPE from January $1^{s t}, 2016$ to December $31^{s t}, 2020$.

\subsection{Event selection}

Data collected in the SAA region are excluded to make sure DAMPE works in a good condition. The following selection criteria are applied in order to select the events crossing all sub-detectors with a proper shower profile development in BGO calorimeter and precise track reconstruction in STK.

- Pre-selection

The first one consists of a set of geometrical fiducial cuts which aim to select the most reliable track: the event must be contained in the sensitive volume from the top PSD Layer to the bottom BGO Layer. Then, for BGO sub-detector, the track must intercept both top and bottom layers within $280 \mathrm{~mm}$ from the respective center. Moreover, the energy deposit is required to be greater than $100 \mathrm{GeV}$ to avoid geomagnetic rigidity cutoff effects for carbon and oxygen.

- Shower profile selection

This selection relies on BGO calorimeter, which is responsible for the energy measurement. The calorimeter is composed of 308 BGO crystal bars $\left(2.5 \times 2.5 \times 60 \mathrm{~cm}^{3}\right)$. The crystals are arranged horizontally in 14 layers, each layer made by 22 bars. The bars of a layer are orthogonal to those of the subsequent one in order to reconstruct the shower profile in both views.

The present analysis step is mainly addressed to select the events with a proper shower development in BGO calorimeter. Firstly, the events are required to pass the High Energy (HE) trigger, which is one of five group trigger logics of the DAMPE detector[10]. HE trigger is triggered when a particle has significant energy deposit in the first four layers of the calorimeter. Then, the energy ratio of the maximum layer energy to the total BGO energy 
must be greater than $0.3+0.2 /\left[1 / \log \left(B G O_{E}\right)\right]$ with the aim of removing the events with too much energy leak at the bottom of $\mathrm{BGO}$ calorimeter, and $B G O_{E}$ is the total deposited energy in BGO calorimeter. Lastly, the maximum energy bar of each layer is required not to be in the first and last two BGO columns in order to remove the events with energy loss at the side edges.

\section{- Track Selection}

The track-finding algorithm typically reconstructs more tracks in the STK, which are caused by pre-showering of cosmic ray and back scattering of the secondary particles. Some selection cuts are used to select the most reliable track: 1) the value of $\chi^{2} / n d o f$ should be less than $150 ; 2)$ the BGO bar hit by the track should be the maximum deposited energy bar of the layer; 3) the PSD strip hit by the track should also be that with the maximum energy deposit one of the two adjacent PSD strips; 4) the energy in the STK cluster hit by track at the first layer should be the maximum energy in the layer; 4) the distance of the candidate track to the BGO track should less than $25 \mathrm{~mm}$; 5) lastly, the ADC value of the STK cluster hit by the track on the first layer should be greater than 500 in order to reduce the contamination of proton and helium.

- Charge selection

The charge measurement is mainly provided by the PSD which is arranged in a double layer configuration and has in total 82 detector strips. The two PSD layers are placed in a hodoscopic configuration. The deposit energy in a hit strip due to ionization is proportional to the square of the particle charge, and is about $2 \mathrm{MeV}$ for a proton crossing vertically the strip. The results of the measured charge correlation between the two PSD layers are shown in Fig. 3 before and after track selection. The charge selection requires that the difference of the charges measured by the two layers must should be less than 1, then the combined charge, which is equal to the average charge of two layers, is used for the analysis. Figure 4 shows the charge spectrums after the different cuts, where the carbon and oxygen peaks can be clearly seen after the final charge selection. Several comparisons between MC data and flight data have also been done for carbon and oxygen, in order to validate the event selection criteria. Figure 5 shows the comparison between $\mathrm{MC}$ and flight data, concerning the behavior of the peak and width of the carbon charge spectrum as a function of the BGO deposited energy. Figure 6 reports the results of the template fits for the BGO energy intervals from $562 \mathrm{GeV}$ to $749 \mathrm{GeV}$ and from $3.2 \mathrm{TeV}$ to $5.6 \mathrm{TeV}$, showing the agreement between $\mathrm{MC}$ data and flight data.

\subsection{High Energy Trigger Efficiency Validation}

The validation of HE trigger efficiency can be checked using the simulation data. Beside the specific cuts (HE trigger), further cuts have been implemented in DAMPE to calculate the efficiency.

Concerning the HE trigger efficiency, it can be estimated in the following way:

$$
\epsilon_{\text {trigger }}=\frac{N_{H E \& L E}}{N_{L E}}
$$



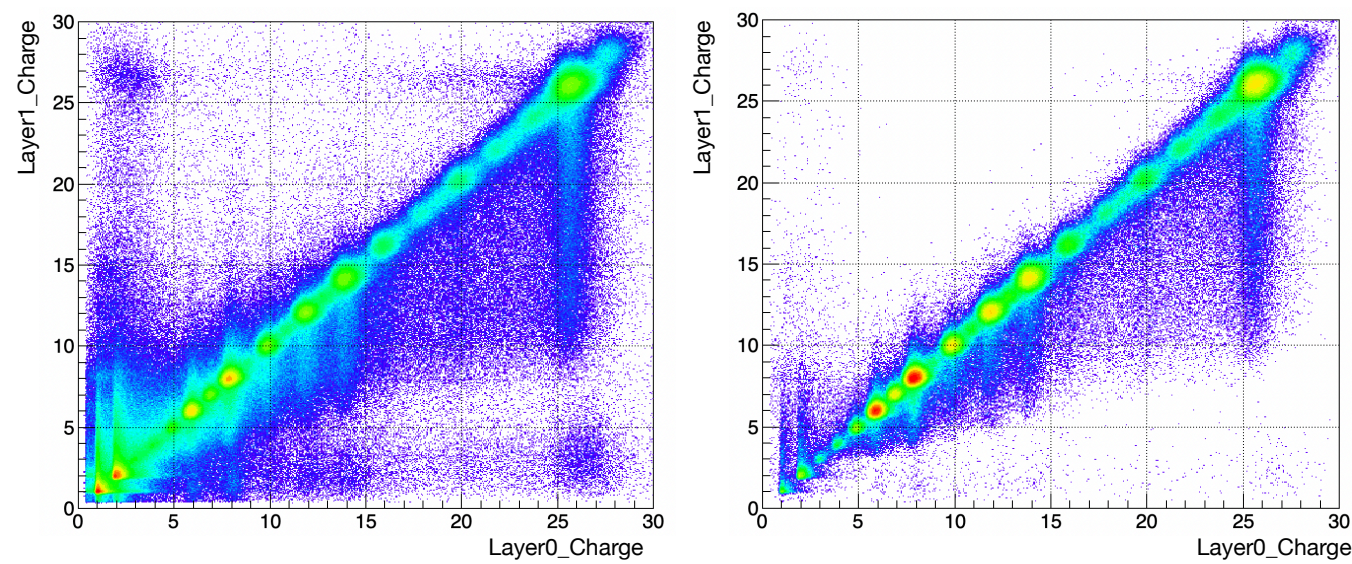

Figure 3: The charge correlation between the two PSD layers, the left one is before track selection and the right one is after track selction

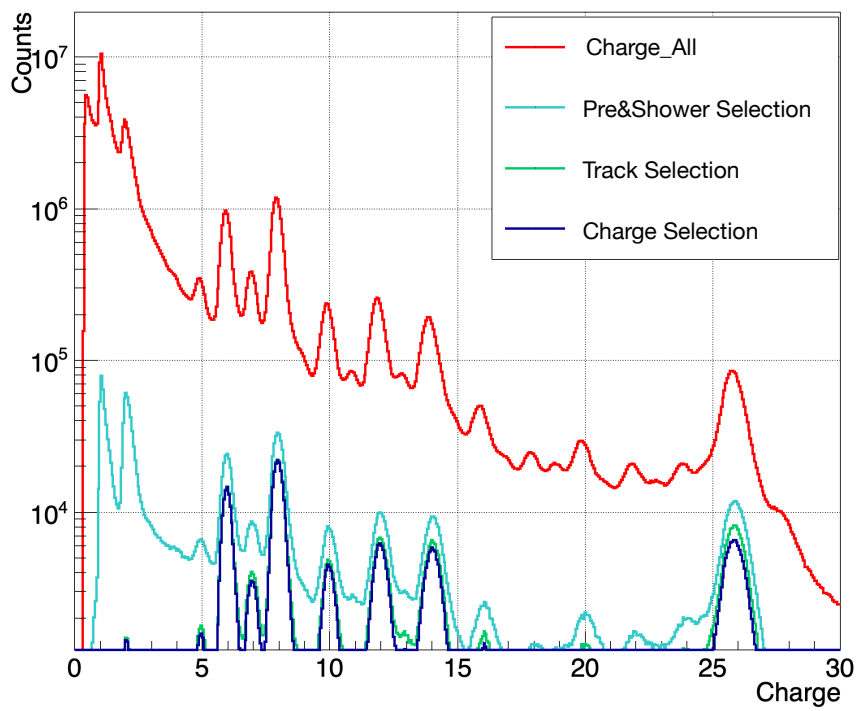

Figure 4: Spectrum of the combined charge from the two PSD layers (see text) at the different selection steps. A significant improvement of the resolution is clearly obtained after the final charge selection (blue line).

where LE refers to the Low Energy trigger, which requires that the particles should pass through the first and the last four BGO layers with low energy deposited. In practice, it is estimated by means of low energy trigger sample, which is pre-scaled by a factor $1 / 8$ at low latitudes $(<20 \mathrm{deg})$ and $1 / 64$ at high latitudes. The results are shown in Fig. 10 ((a) and (b)), from which we can see that the efficiency for data is $100 \%$ when the deposited energy is above $400 \mathrm{GeV}$. Because of the pre-scale factor applied to low energy trigger, the statistics is limited at high energy. From the result, the HE trigger efficiency of flight data is in good agreement with MC simulation, the difference being less than $2 \%$ for both carbon and oxygen. 

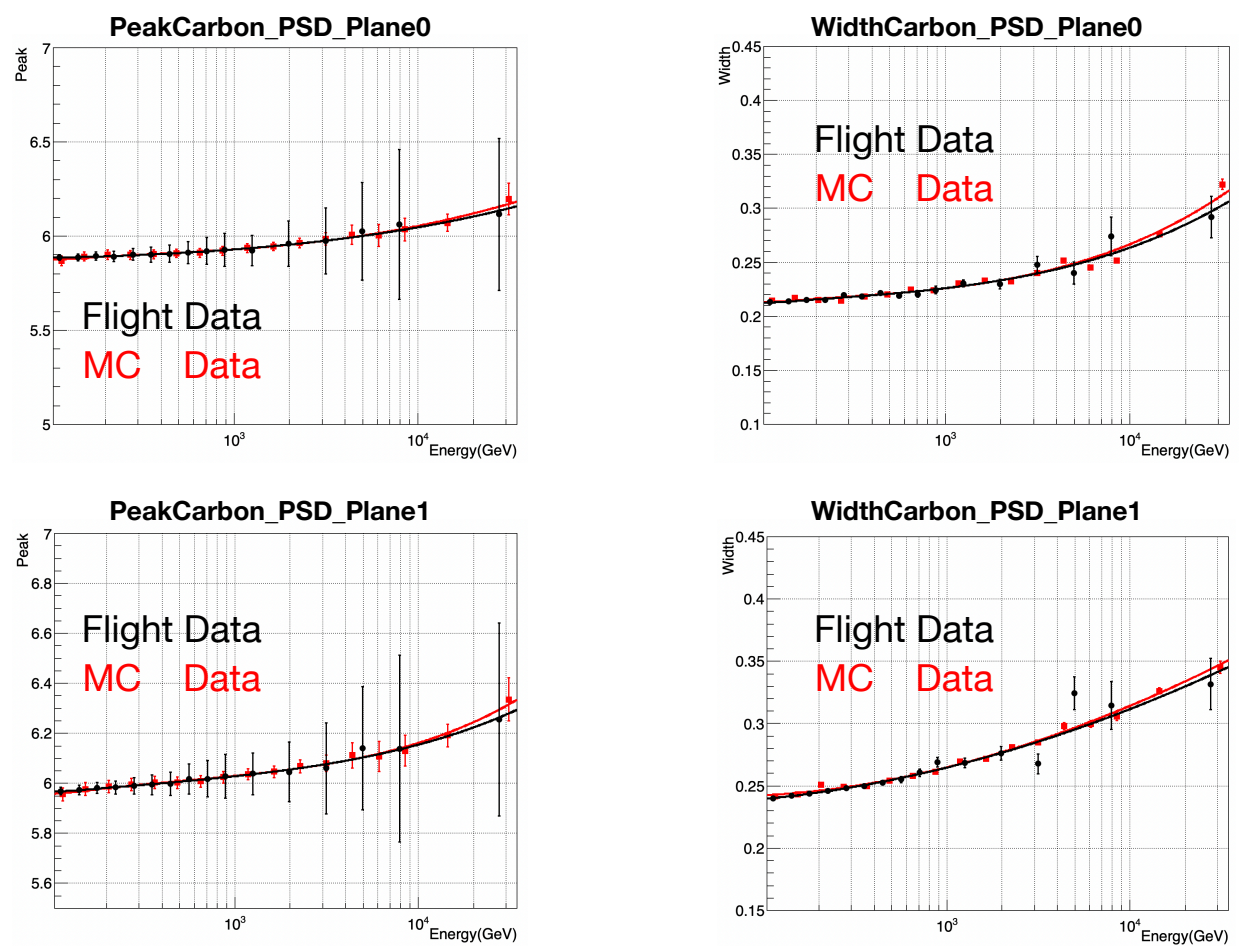

Figure 5: The comparison between flight data and Monte Carlo data of both peak and width for Carbon charge spectrum, as a function of BGO deposited energy. The top plots refer to the results of charge peak and width for one layer, the bottom plots for the other layer.
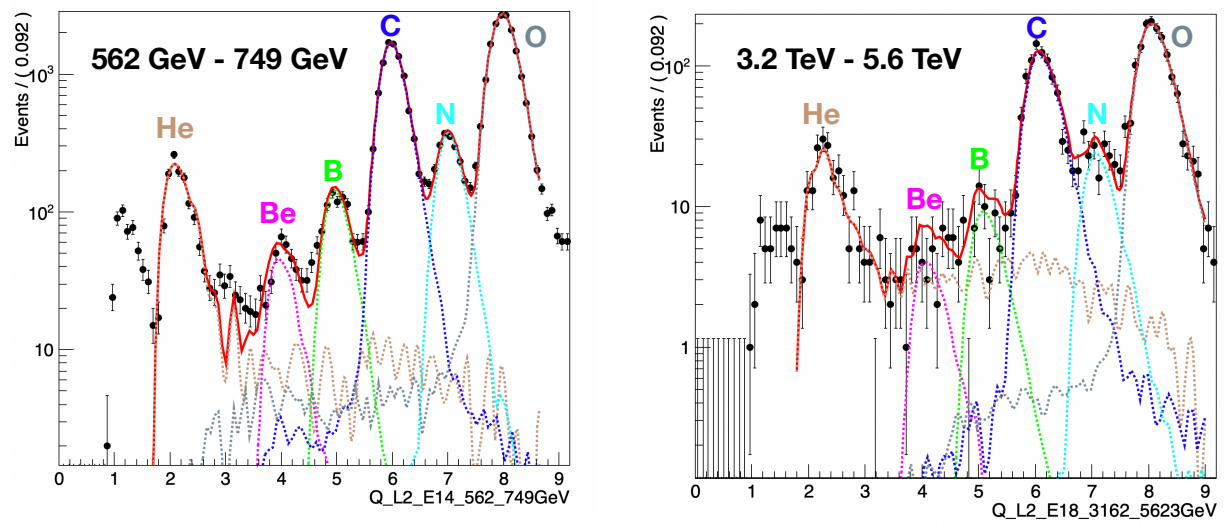

Figure 6: The results of template fit. The left one is the result for the range from $562 \mathrm{GeV}$ to $749 \mathrm{GeV}$, the right one for the range from $3.2 \mathrm{TeV}$ to $5.6 \mathrm{TeV}$.

\section{Conclusion}

DAMPE has been in smooth operation for more than 5 years since its launch on Dec. 17th 2015. Some criteria for selecting carbon and oxygen events were developed, and the charge of carbon and oxygen after final cut were validated by Monte Carlo data, some efficiencies of selection criteria were also validated by Monte Carlo data. 

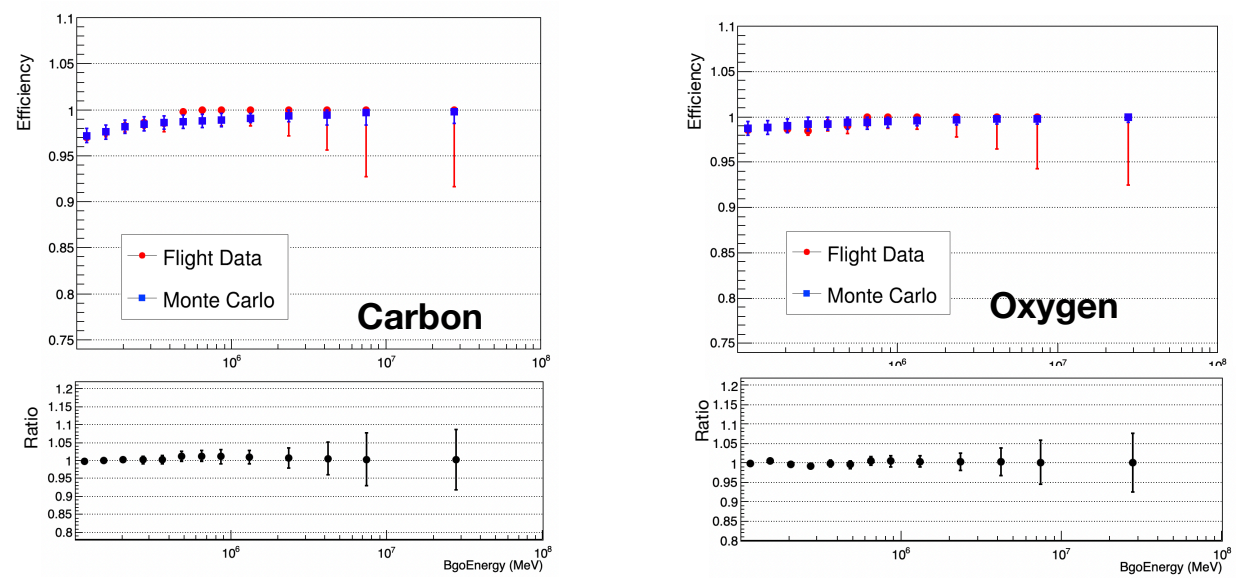

Figure 7: HE trigger efficiency as a function of the energy deposited in BGO calorimeter (top figures) and difference between simulation and flight data expressed by the ratio between the related efficiencies as a function of deposited energy (bottom figures). The left plot is the result for carbon, and the right one for oxygen.

More validation tests with different simulation models and more precise evaluations of systematic uncertainties are necessary for next analysis steps.

\section{References}

[1] Aguilar M, Aisa D, Alvino A, et al. Electron and positron fluxes in primary cosmic rays measured with the Alpha Magnetic Spectrometer on the International Space Station[J]. Physical Review Letters, 2014, 113(12): 121102.

[2] Adriani O, Barbarino G C, Bazilevskaya G A, et al. Cosmic-ray electron flux measured by the PAMELA experiment between 1 and $625 \mathrm{GeV}[\mathrm{J}]$. Physical Review Letters, 2011, 106(20): 201101.

[3] Niita T, Torii S, Akaike Y, et al. Energy calibration of Calorimetric Electron Telescope (CALET) in space[J]. Advances in Space Research, 2015, 55(11): 2500-2508.

[4] Abdo A A, Ackermann M, Ajello M, et al. The on-orbit calibration of the Fermi Large Area Telescope[J]. Astroparticle Physics, 2009, 32(3): 193-219.

[5] Chang J, Ambrosi G, An Q, et al. The DArk Matter Particle Explorer mission[J]. Astroparticle Physics, 2017, 95: 6-24.

[6] Ambrosi G, An Q, Asfandiyarov R, et al. The on-orbit calibration of DArk Matter Particle Explorer[J]. Astroparticle Physics, 2019, 106: 18-34.

[7] Y. Yu et al, arXiv:1703.00098

[8] P. Azzarello et al., Nucl. Instrum. Meth. A 831 (2016) 378-384 
[9] Wu L, Wen S, Liu C, et al. Calibration and Status of the 3-D Imaging Calorimeter of DAMPE for Cosmic Ray Physics on Orbit[J]. IEEE Transactions on Nuclear Science, 2018, 65(8): 2007-2012.

[10] Zhang Y Q, Guo J H, Liu Y, et al. Design and on-orbit status of the trigger system for the DAMPE mission[J]. Research in Astronomy and Astrophysics, 2019, 19(9): 123. 


\section{Full Authors List: DAMPE* Collaboration}

F. Alemanno ${ }^{1,2}$, Q. An ${ }^{3,4}$, P. Azzarello ${ }^{5}$, F. C. T. Barbato ${ }^{1,2}$, P. Bernardini ${ }^{6,7}$, X. J. Bi ${ }^{8,9}$, M. S. Cai ${ }^{10,11}$, E. Casilli6, ${ }^{6,}$ E. Catanzani ${ }^{12}$, J. Chang ${ }^{10,11}$, D. Y. Chen ${ }^{10}$, J. L. Chen ${ }^{13}$, Z. F. Chen ${ }^{10,11}$, M. Y. Cui ${ }^{10}$, T. S. Cui ${ }^{14}$, Y. X. Cui ${ }^{10,11}$, H. T. Dai ${ }^{3,4}$, A. De Benedittis ${ }^{6,7}$,

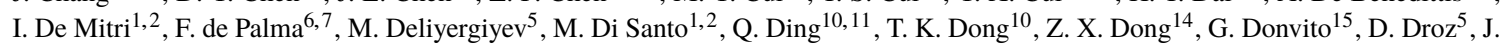
L. Duan ${ }^{13}$, K. K. Duan ${ }^{10}$, D. D’Urso ${ }^{12, \dagger}$, R. R. Fan ${ }^{8}$, Y. Z. Fan ${ }^{10,11}$, K. Fang ${ }^{8}$, F. Fang ${ }^{13}$, C. Q. Feng ${ }^{3,4}$, L. Feng ${ }^{10}$, P. Fusco ${ }^{15,16}$, M. $\mathrm{Gao}^{8}$, F. Gargano ${ }^{15}$, K. Gong ${ }^{8}$, Y. Z. Gong ${ }^{10}$, D. Y. Guo ${ }^{8}$, J. H. Guo ${ }^{10,11}$, S. X. $\mathrm{Han}^{14}$, Y. M. Hu ${ }^{10}$, G. S. Huang ${ }^{3,4}$, X. Y. Huang ${ }^{10,11}$, Y. Y. Huang ${ }^{10}$, M. Ionica ${ }^{12}$, W. Jiang ${ }^{10}$, J. Kong ${ }^{13}$, A. Kotenko ${ }^{5}$, D. Kyratzis ${ }^{1,2}$, S. J. Lei ${ }^{10}$, W. H. Li ${ }^{10,11}$, W. L. Li ${ }^{14}$, X. Li ${ }^{10}$, X. Q.

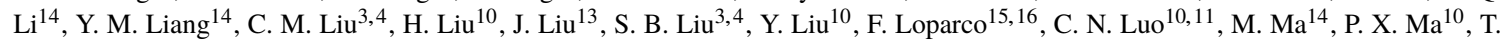

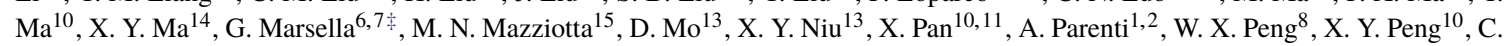
Perrina $^{5,8}$, R. Qiao ${ }^{8}$, J. N. Rao ${ }^{14}$, A. Ruina ${ }^{5}$, M. M. Salinas ${ }^{5}$, G. Z. Shang ${ }^{14}$, W. H. Shen ${ }^{14}$, Z. Q. Shen ${ }^{10}$, Z. T. Shen ${ }^{3,4}$, L. Silveri ${ }^{1,2}$, J. X. Song ${ }^{14}$, M. Stolpovskiy ${ }^{5}$, H. Su ${ }^{13}$, M. Su ${ }^{17}$, H. R. Sun ${ }^{3,4}$, Z. Y. Sun ${ }^{13}$, A. Surdo ${ }^{7}$, X. J. Teng ${ }^{14}$, A. Tykhonov ${ }^{5}$, H. Wang ${ }^{14}$, J. Z.

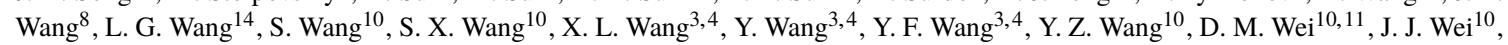
Y. F. $\mathrm{We}^{3,4}$, D. $\mathrm{Wu}^{8}$, J. Wu ${ }^{10,11}$, L. B. $\mathrm{Wu}^{1,2}$, S. S. $\mathrm{Wu}^{14}$, X. Wu $\mathrm{Wu}^{5}$ Z. Q. Xia ${ }^{10}$, H. T. Xu ${ }^{14}$, Z. H. Xu ${ }^{10,11}$, Z. L. Xu ${ }^{10}$, E. H. Xu $u^{3,4}, Z$. Z. Xu ${ }^{3,4}$, G. F. Xue ${ }^{14}$, H. B. Yang ${ }^{13}$, P. Yang ${ }^{13}$, Y. Q. Yang ${ }^{13}$, H. J. Yao ${ }^{13}$, Y. H. Yu ${ }^{13}$, G. W. Yuan ${ }^{10,11}$, Q. Yuan ${ }^{10,11}$, C. Yue ${ }^{10}$, J. J. Zang $^{10 \pi}$ S. X. Zhang ${ }^{13}$, W. Z. Zhang ${ }^{14}$, Yan Zhang ${ }^{10}$, Yi Zhang ${ }^{10,11}$, Y. J. Zhang ${ }^{13}$, Y. L. Zhang ${ }^{3,4}$, Y. P. Zhang ${ }^{13}$, Y. Q. Zhang ${ }^{10}, Z$. Zhang $^{10}$, Z. Y. Zhang ${ }^{3,4}$, C. Zhao ${ }^{3,4}$, H. Y. Zhao ${ }^{13}$, X. F. Zhao ${ }^{14}$, C. Y. Zhou ${ }^{14}$, and Y. Zhu ${ }^{14}$

${ }^{1}$ Gran Sasso Science Institute (GSSI), Via Iacobucci 2, I-67100 L'Aquila, Italy

${ }^{2}$ Istituto Nazionale di Fisica Nucleare (INFN) -Laboratori Nazionali del Gran Sasso, I-67100 Assergi, L'Aquila, Italy

${ }^{3}$ State Key Laboratory of Particle Detection and Electronics, University of Science and Technology of China, Hefei 230026, China

${ }^{4}$ Department of Modern Physics, University of Science and Technology of China, Hefei 230026, China

${ }^{5}$ Department of Nuclear and Particle Physics, University of Geneva, CH-1211, Switzerland

${ }^{6}$ Dipartimento di Matematica e Fisica E. De Giorgi, Universita' del Salento, I-73100, Lecce, Italy

${ }^{7}$ Istituto Nazionale di Fisica Nucleare (INFN) - Sezione di Lecce, I-73100, Lecce, Italy

${ }^{8}$ Institute of High Energy Physics, Chinese Academy of Sciences, Yuquan Road 19B, Beijing 100049, China

${ }^{9}$ University of Chinese Academy of Sciences, Yuquan Road 19A, Beijing 100049, China

${ }^{10}$ Key Laboratory of Dark Matter and Space Astronomy, Purple Mountain Observatory, Chinese Academy of Sciences, Nanjing 210023, China

${ }^{11}$ School of Astronomy and Space Science, University of Science and Technology of China, Hefei 230026, China

${ }^{12}$ Istituto Nazionale di Fisica Nucleare (INFN) - Sezione di Perugia, I-06123 Perugia, Italy

${ }^{13}$ Institute of Modern Physics, Chinese Academy of Sciences, Nanchang Road 509, Lanzhou 730000, China

${ }^{14}$ National Space Science Center, Chinese Academy of Sciences, Nanertiao 1, Zhongguancun, Haidian district, Beijing 100190, China

${ }^{15}$ Istituto Nazionale di Fisica Nucleare (INFN) - Sezione di Bari, I-70125, Bari, Italy

${ }^{16}$ Dipartimento di Fisica “M. Merlin" dell'Universita“ e del Politecnico di Bari, I-70126, Bari, Italy

${ }^{17}$ Department of Physics and Laboratory for Space Research, the University of Hong Kong, Pok Fu Lam, Hong Kong SAR, China

\footnotetext{
* The DAMPE mission is funded by the Strategic Priority Science and Technology Projects in Space Science of the Chinese Academy of Sciences. This work is supported by the National Key Research and Development Program of China (2016YFA0400200 and 2016YFA0400202), the Project supported by the Joint Funds of the National Natural Science Foundation of China (No. U1738208, U1738139 and U1738135) and the National Natural Science Foundation of China (No. 11673021 and No. 11705197). In Europe the activities and the data analysis are supported by the Swiss National Science Foundation (SNSF), Switzerland; the National Institute for Nuclear Physics (INFN), Italy.

${ }^{\dagger}$ Now at Dipartimento di Chimica e Farmacia, Universita' di Sassari, I-07100, Sassari, Italy.

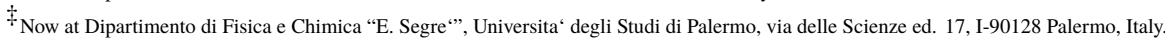

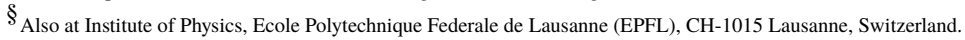

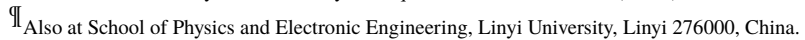

